\title{
A Comparison Between Malignant Breast Neoplasms Apoptosis and Benign Breast Neoplasms Under Influence of DNA Fragmentation
}

\author{
Parisa Derogar ${ }^{1}$, Ali Akbar Abolfathi ${ }^{1}$, Asal Safi Samghabadi ${ }^{2}$, \\ Afsaneh Yazdani ${ }^{1}$, Fateme Afkhami ${ }^{3,}{ }^{*}$ \\ ${ }^{1}$ Biochemical Department, Ahar Branch, Islamic Azad University, Ahar, Iran \\ ${ }^{2}$ Cell and molecular Biology Department, Tehran Branch, Islamic Azad University, \\ Science and Research Branch, Tehran, Iran \\ ${ }^{3}$ Department of Medical Genetics, Faculty of Medicine, Tabriz University of Medical \\ Sciences, Tabriz, Iran \\ ${ }^{*}$ Corresponding Author: Fateme Afkhami, Medical Genetic Department, \\ Medical Faculty, Tabriz University of Medical Sciences, Tabriz, Iran. E-mail: \\ fatemeafkhamil@yahoo.com
}

DOI: $10.21859 / \mathrm{mci}$-supp-16

\section{Keywords:}

DNA Fragmentation

Apoptosis

Breast Cancer

Benign

Malignant

\begin{abstract}
Introduction Interrelationship of cells proliferation and apoptosis regulate homeostasis in normal tissues. Breast cancer development is mainly due to defective apoptosis pathway. Since there is a significant interrelationship between the ratio of apoptotic bodies to normal cells and breast cancer pathogenesis, we conducted this study to contrast malignant breast neoplasms apoptosis with benign breast neoplasms under the influence of DNA Fragmentation.

Materials and Methods: Sixty patients entered this study. Its control group were their both benign neoplasms and normal cells. After DNA extraction, DNA fragmentation was carried out with DNA laddering assay. Apoptotic bodies were detected in $1 \%$ agarose gel electrophoresis.

Results: Apoptotic bodies were detected in $83.3 \%$ of breast cancer samples. However, no sign of apoptosis was observed in control samples and the number of positive cases in malignant samples was significantly higher than benign samples $(\mathrm{P}<0.05)$.

Conclusions: It is revealed that the rate of apoptosis detectable by DNA fragmentation in malignant breast neoplasms is higher than benign types so that it can be used as a quick-diagnostic technique.
\end{abstract}

